\title{
A NOTE ON THE STABILITY AND THE APPROXIMATION OF SOLUTIONS FOR A DIRICHLET PROBLEM WITH $p(x)$-LAPLACIAN
}

\author{
MAREK GALEWSKI ${ }^{1}$
}

(Received 1 September 2006; revised 11 June 2007)

\begin{abstract}
We show the stability results and Galerkin-type approximations of solutions for a family of Dirichlet problems with nonlinearity satisfying some local growth conditions.

2000 Mathematics subject classification: primary 35A 15; secondary 35B35, 65N30.

Keywords and phrases: $p(x)$-Laplacian, duality, variational method, stability of solutions, Galerkin-type approximations.
\end{abstract}

\section{Introduction}

The aim of this paper is to consider the existence and stability of solutions and their Galerkin-type finite-dimensional approximations for the family of Dirichlet problems given by

$$
\begin{aligned}
& -\operatorname{div}\left(a_{k}(x)|\nabla u(x)|^{p(x)-2} \nabla u(x)\right)=F_{u}^{k}(x, u(x)), \\
& \left.u(x)\right|_{\partial \Omega}=0, \quad u \in W_{0}^{l, p(x)}(\Omega)
\end{aligned}
$$

where $\Omega \subset R^{N}$ is a bounded region with Lipschitz boundary, $p, q \in C(\bar{\Omega})$ and $1 / p(x)+1 / q(x)=1$ for $x \in \Omega ; W_{0}^{1, p(x)}(\Omega)$ denotes the generalized Orlicz-Sobolev space, see [6, 7]; and $a_{k} \in C(\bar{\Omega})$ with $a_{0}(x) \geq a_{0}>0$ on $\bar{\Omega}$ for $k=0,1,2, \ldots$ Let $p^{-}=\inf _{x \in \Omega} p(x)>N>2, a_{k} \rightrightarrows a_{0}$.

The stability of the system (1.1) means that for each $k=1,2, \ldots$ there exists a solution $u_{k}$ to (1.1) and there exists $\bar{u}=\lim _{k \rightarrow \infty} u_{k}$ (weak in $W_{0}^{1, p(x)}(\Omega)$ ) solving

$$
\begin{aligned}
& -\operatorname{div}\left(a_{0}(x)|\nabla \bar{u}(x)|^{p(x)-2} \nabla u(x)\right)=F_{u}^{0}(x, \bar{u}(x)), \\
& \left.\bar{u}(x)\right|_{\partial \Omega}=0, \quad \bar{u} \in W_{0}^{1, p(x)}(\Omega) .
\end{aligned}
$$

\footnotetext{
'Faculty of Mathematics and Computer Science, University of Lodz, Banacha 22, 90-238 Lodz, Poland; email: galewski@math.uni.lodz.pl.

(C) Australian Mathematical Society 2007, Serial-fee code 1446-1811/07
} 
The idea of the approximation of solutions is based on the observation that each $u_{k}$ solving (1.1) is approximated by a suitably selected minimizing sequence $\left\{u_{k}^{n}\right\}_{n=1}^{\infty}$ for the action functional $J_{k}: W_{0}^{1, p(x)}(\Omega) \rightarrow R$

$$
J_{k}(u)=\int_{\Omega} \frac{a_{k}(x)}{p(x)}|\nabla u(x)|^{p(x)} d x-\int_{\Omega} F^{k}(x, u(x)) d x .
$$

We minimize $J_{k}$ on a set $X_{k}$ which has the following property: for all $u \in X_{k}$, the relation

$$
\begin{aligned}
& -\operatorname{div}\left(a_{k}(x)|\nabla \tilde{u}(x)|^{p(x)-2} \nabla \tilde{u}(x)\right)=F_{u}^{k}(x, u(x)), \\
& \left.\tilde{u}(x)\right|_{\partial \Omega}=0, \quad \tilde{u} \in W_{0}^{1, p(x)}(\Omega)
\end{aligned}
$$

implies $\tilde{u} \in X_{k}$. We later show that the minimizing sequence $\left\{u_{k}^{n}\right\}_{n=1}^{\infty}$ is such that

$$
-\operatorname{div}\left(a_{k}(x)\left|\nabla u_{k}^{n}(x)\right|^{p(x)-2} \nabla u_{k}^{n}(x)\right)=F_{u}^{k}\left(x, z_{k}^{n}(x)\right),
$$

where $z_{k}^{n}$ for each $n=1,2, \ldots$ is a certain element of $X_{k}$. Thus each $u_{k}^{n}$ - being a solution to Equation (1.4) which has a fixed right-hand side - may be approximated by a Galerkin sequence. Consult [2] for details of Galerkin-type approximations for equations in Orlicz-Sobolev space, and Section 4. Now a solution is approximated by a minimizing sequence, whose elements in turn may be approximated by a sequence of finite-dimensional elements. Therefore by taking a diagonal sequence we obtain the sequence of finite-dimensional solutions to (1.2). The very same idea applies to approximation of the solution of (1.2).

Concerning the existence and stability of solutions with the use of a dual variational method we draw attention to our previous paper [8]. The following Dirichlet problem is investigated:

$$
\begin{aligned}
& -\operatorname{div}\left(a(x)|\nabla u(x)|^{p(x)-2} \nabla u(x)\right)+b(x)|u(x)|^{p(x)-2} u(x)=F_{u}^{k}(x, u(x)), \\
& \left.u(x)\right|_{\partial \Omega}=0, \quad u \in W_{0}^{1, p(x)}(\Omega),
\end{aligned}
$$

where $a, b \in L^{\infty}(\Omega)$ with $a(x) \geq a_{0}>0$ and $b(x) \geq b_{0} \geq 0$ a.e. on $\Omega$ and with nonlinearity satisfying some local conditions being counterparts of our conditions 1-3 (see Section 2). In reaching the existence result one finds a set on which one may minimize the action functional for which (1.5) is the Euler-Lagrange equation. Later, a suitable minimizing sequence is chosen and its convergence investigated. In order to demonstrate that the limit of a minimizing sequence is actually a solution to (1.5), a duality theory is constructed. In doing so a dual action functional is introduced, its domain is constructed and both action functionals are minimized. Finally, the existence results are used to obtain stability of solutions to (1.5) in the sense described above. Galerkin-type approximations have not been considered in [8]. 
In the present paper we are concerned with the case when the function $a(x)$ from (1.5) may also vary with a numerical parameter $k$. In order to investigate the stability and approximation of solutions one should have suitable existence results first. Since in proving the existence result we find a minimizing sequence, there arose a question if any minimizing sequence - whose existence on a set $X_{k}$ follows by the growth conditions - will allow us to obtain the approximation result. It appeared that property (1.4) for a minimizing sequence was required. Therefore we use some ideas from [8] concerning the construction of a dual action functional $J_{D}^{k}$ with suitable modifications for selecting the minimizing sequence for $J_{k}$ over $X_{k}$. In order to get property (1.4) we first find a minimizing sequence for a dual functional and later construct a suitable minimizing sequence for $J_{k}$ over $X_{k}$. The present paper and paper [8] differ as far as the existence result is concerned in their approach towards selecting minimizing sequences. The existence of a minimizing sequence for $J_{D}^{k}$ follows by the growth conditions which we use and the construction of the sets on which a dual action functional is minimized, while in [8] the action functional $J_{k}$ was minimized. However, since the minimizing sequence for $J_{k}$ over $X_{k}$ must have property (1.4) we must use additional arguments and the form of proof used in [8] does not apply here.

For some other variational approaches see [3] and [5], where the existence of a weak solution to a $p(x)$-Laplacian equation is considered. In both sources the direct variational method is used in order to get solutions in the sublinear case while the Palais-Smale condition is imposed and mountain pass geometry is employed to get the solution in the superlinear case.

Since problems with generalized-growth conditions are applied in elastic mechanics and electrorheological fluid dynamics, see $[10,11]$ and references therein, we believe that our results may contribute to that research.

\section{The assumptions and auxiliary results}

In what follows we denote the best Sobolev constant by $C_{S}$, where

$$
\|u\|_{p(x)} \leq C_{S}\|\nabla u\|_{p(x)} \quad \text { for all } u \in W_{0}^{1, p(x)}(\Omega ; R) .
$$

Since $W_{0}^{1, p(x)}(\Omega ; R)$ is continuously embedded into $W_{0}^{1, p^{-}}(\Omega)$, [7], we have

$$
\|\nabla u\|_{p^{-}} \leq C_{1}\|\nabla u\|_{p(x)}
$$

for some constant $C_{1}$. Since $p^{-}>N$, by the Sobolev embedding theorem [1] we get

$$
\max _{x \in \Omega}|u(x)| \leq C_{2}\|\nabla u\|_{p^{-}} \quad \text { for all } u \in W_{0}^{1, p}(\Omega)
$$

for a certain constant $C_{2}$. Relation (2.2) is understood as follows: for every $u \in W_{0}^{1, p}(\Omega)$ there exists $u_{0} \in C(\Omega)$ such that $u_{0}(x)=u(x)$ almost everywhere and $\max _{x \in \Omega}\left|u_{0}(x)\right| \leq C_{2}\|\nabla u\|_{\rho}$. 
Therefore by (2.1) and (2.2) for all $u \in W_{0}^{1, p(x)}(\Omega ; R)$ we get

$$
\max _{x \in \Omega}|u(x)| \leq C_{2}\|\nabla u\|_{p^{-}} \leq C_{1} C_{2}\|\nabla u\|_{p(x)} .
$$

We assume that the following hold for a function $F^{k}$.

Assumption 1. The relation $\|1\|_{q(x)}\left(1 / p^{-}+1 / q^{-}\right)^{-1} \leq 1$ is satisfied; there exist positive numbers $d_{0}, d_{1}, d_{2}, \ldots$ such that $d_{k} \leq d_{0}$ for $k \in N$ and

$$
\begin{aligned}
& C_{S} \underset{x \in \Omega}{\operatorname{ess} \sup }\left|F_{u}^{k}\left(x, d_{k}\right)\right| \geq 1, \quad C_{S} \underset{x \in \Omega}{\operatorname{ess} \sup }\left|F_{u}^{k}\left(x,-d_{k}\right)\right| \geq 1, \\
& C_{S} C_{1} C_{2} \text { ess sup }\left|F_{u}^{k}\left(x, d_{k}\right)\right| \leq a_{0} d_{k}, \quad C_{S} C_{1} C_{2} \text { ess sup }\left|F_{u}^{k}\left(x,-d_{k}\right)\right| \leq a_{0} d_{k} .
\end{aligned}
$$

Assumption 2. There exists a positive number $d>d_{0}$ such that for all $k=$ $0,1,2, \ldots$ and $I=[-d, d]: F_{u}^{k}(\cdot, d), F_{u}^{k}(\cdot,-d) \in L^{\infty}(\Omega), F^{k}: \Omega \times I \rightarrow R$ are Caratheodory functions and convex in $u$ for a.e. $x \in \Omega, F_{u}^{k}: \Omega \times I \rightarrow R$ are Caratheodory functions and $F^{k}(x, u):=+\infty$ for $(x, u) \in \Omega \times(R-I)$.

Assumption 3. The function $F_{u}^{k}(x, 0) \neq 0$, for a.e. $x \in \Omega, x \longmapsto F^{k}(x, 0)$ and $x \longmapsto\left(F^{k}\right)^{*}(x, 0)$ are integrable on $\Omega$.

Here $\left(F^{k}\right)^{*}$ denotes the Fenchel-Young conjugate of $F^{k}$, see [4]. Now it is obvious that $F^{k}: \Omega \times R \rightarrow R$ is convex and l.s.c.

Let $W=\left\{v \in L^{q(x)}(\Omega) \mid \operatorname{div} v \in L^{q(x)}(\Omega)\right\}$. The dual functional which we investigate reads $J_{k}^{D}: W \rightarrow R$

$$
J_{k}^{D}(v)=\int_{\Omega}\left(F^{k}\right)^{*}(x,-\operatorname{div} v(x)) d x-\int_{\Omega} \frac{1}{\left(a_{k}(x)\right)^{q(x) / p(x)}} \frac{1}{q(x)}|v(x)|^{q(x)} d x .
$$

We put

$$
X_{k}=\left\{u \in W_{0}^{1, p(x)}(\Omega), \quad\|\nabla u\|_{p(x)} \leq \frac{d_{k}}{C_{1} C_{2}}, \quad|u(x)| \leq d_{k}\right\}
$$

and exactly as in [8] we prove that this set has property (1.3).

The dual functional $J_{k}^{D}$ will be considered on a set $X_{k}^{d}$ which is a set of these $v \in W$ for which there exists $a u \in X_{k}$ such that

$$
-\operatorname{div} v(x)=F^{k}(x, u(x))
$$

and

$$
a_{k}(x)|\nabla \tilde{u}(x)|^{p(x)-2} \nabla \tilde{u}(x)=v(x),
$$

where $\tilde{u}$ corresponds to $u$ in (1.3).

We will investigate $J_{k}$ on a set $X_{k}$ and $J_{k}^{D}$ on a set $X_{k}^{d}$ on which sets these are well defined. 


\section{The existence and stability of solutions}

THEOREM 3.1. Assume Assumptions 1,2 and 3 hold. For all $k=0,1,2, \ldots$ there exists $\left(u_{k}, v_{k}\right) \in X_{k} \times X_{k}^{d}$ such that

$$
\begin{gathered}
-\operatorname{div} v_{k}(x)=F_{u}^{k}\left(x, u_{k}(x)\right) \quad \text { and } \\
a_{k}(x)\left|\nabla u_{k}(x)\right|^{p(x)-2} \nabla u_{k}(x)=v_{k}(x) .
\end{gathered}
$$

Moreover

$$
\inf _{v \in X_{k}^{d}} J_{k}^{D}(v)=J_{k}^{D}\left(v_{k}\right)=J_{k}\left(u_{k}\right)=\inf _{u \in X_{k}} J_{k}(u)
$$

PROOF. We fix $k=0,1,2, \ldots$ We observe that $\inf _{u \in X_{k}} J_{k}(u)$ is finite by Lemma 3.4 of [8]. Exactly as in [8] we can show that

$$
\inf _{v \in X_{k}^{d}} J_{k}^{D}(v)=\inf _{u \in X_{k}} J_{k}(u)
$$

By (3.4) since $\inf _{u \in X_{k}} J_{k}(u)$ is finite it follows that $J_{k}^{D}$ is bounded from below on $X_{k}^{d}$. By the definition of $X^{d}$, see (2.3), by the properties of the Fenchel-Young transform it follows that there exists a constant $\eta_{k}>0$ such that $\int_{\Omega}\left(F^{k}\right)^{*}(x,-\operatorname{div} v(x)) d x \leq \eta_{k}$ for all $v \in X_{k}^{d}$. Therefore by the definition of $J_{k}^{D}$ and since $J_{k}^{D}(v) \geq \inf _{u \in X_{k}} J_{k}(u)$ for all $v \in X_{k}^{d}$ we get

$$
\begin{aligned}
\int_{\Omega} \frac{1}{\left(a_{k}(x)\right)^{q(x) / p(x)}} \frac{1}{q(x)}|v(x)|^{q(x)} d x & \leq \int_{\Omega}\left(F^{k}\right)^{*}(x,-\operatorname{div} v(x)) d x-\inf _{u \in X_{k}} J_{k}(u) \\
& \leq \eta_{k}-\inf _{u \in X_{k}} J_{k}(u) .
\end{aligned}
$$

By (2.3) and by Assumptions 1 and 2 we get for a.e. $x \in \Omega$, for any $h \in X_{k}$ that $F_{u}^{k}\left(x,-d_{k}\right) \leq F_{u}^{k}(x, h(x)) \leq F_{u}^{k}\left(x, d_{k}\right)$. Thus $F_{u}^{k}(\cdot, h(\cdot))$ is bounded in $L^{\infty}(\Omega)$ and so is $\operatorname{div} v(\cdot)$. Since the term $1 /\left(a_{k}(x)\right)^{q(x) / p(x)} \cdot 1 / q(x)$ is bounded, it follows that $X_{k}^{d}$ is bounded in $W$. Thus we may find a minimizing sequence $\left\{v_{k}^{n}\right\}_{n=1}^{\infty}$ for a restriction of a functional $J_{k}^{D}$ to the set $X_{k}^{d}$ which may be assumed to be weakly convergent in $W$.

We show that there exists a minimizing sequence $\left\{u_{k}^{n}\right\}_{n=1}^{\infty}$ for a restriction of functional $J_{k}$ to the set $X_{k}$ having the property for $n=1,2, \ldots$

$$
a_{k}(x)\left|\nabla u_{k}^{n}(x)\right|^{p(x)-2} \nabla u_{k}^{n}(x)=v_{k}^{n}(x)
$$

Since $v_{k}^{n} \in X_{k}^{d}$ it follows that $u_{k}^{n} \in X_{k}$. We observe that for $v_{k}^{n}$ and $u_{k}^{n}$, by (3.5) we 
have by properties of the Fenchel-Young transform that

$$
\begin{aligned}
J_{k}^{D}\left(v_{k}^{n}\right) & =\int_{\Omega}\left(F^{k}\right)^{*}\left(x,-\operatorname{div} v_{k}^{n}(x)\right) d x-\int_{\Omega} \frac{1}{\left(a_{k}(x)\right)^{q(x) / p(x)}} \frac{1}{q(x)}\left|v_{k}^{n}(x)\right|^{q(x)} d x \\
& =\int_{\Omega}\left(F^{k}\right)^{*}\left(x,-\operatorname{div} v_{k}^{n}(x)\right) d x+\int_{\Omega}\left(\operatorname{div} v(x) u_{k}^{n}(x)+\frac{a_{k}(x)}{p(x)}\left|\nabla u_{k}^{n}(x)\right|^{p(x)}\right) d x \\
& \geq \int_{\Omega} \frac{a_{k}(x)}{p(x)}\left|\nabla u_{k}^{n}(x)\right|^{p(x)} d x-\int_{\Omega} F^{k}\left(x, u_{k}^{n}(x)\right) d x=J_{k}\left(u_{k}^{n}\right) .
\end{aligned}
$$

By the above and since $\left\{v_{k}^{n}\right\}_{n=1}^{\infty}$ is a minimizing sequence, we obtain that for any $\varepsilon>0$ there exists $n_{0}$ such that for all $n>n_{0}$ we get

$$
J_{k}\left(x_{k}^{n}\right)+\varepsilon \geq \inf _{u \in X_{k}} J_{k}(u)+\varepsilon \geq J_{k}^{D}\left(v_{k}^{n}\right) \geq J_{k}\left(u_{k}^{n}\right) \geq \inf _{u \in X_{k}} J_{k}(u) .
$$

Thus $\left\{u_{k}^{n}\right\}_{n=n_{0}}^{\infty}$ is a minimizing sequence. We renumber both sequences so that these start with $n=1$. Sequence $\left\{u_{k}^{n}\right\}_{n=1}^{\infty}$ may be assumed to be weakly convergent in $W_{0}^{1, p(x)}(\Omega)$ and therefore, up to a subsequence, strongly in $L^{p^{-}}(\Omega)$. Thus a sequence $\left\{u_{k}^{n}\right\}_{n=1}^{\infty}$ contains a subsequence convergent a.e. We denote this subsequence by $\left\{u_{k}^{n}\right\}_{n=1}^{\infty}$ and its limit by $u_{k}$. We see that $\left\|\nabla u_{k}^{n}\right\|_{L^{p(x)}(\Omega)} \leq d_{k} / C_{1} C_{2}$ for all $n$ and $\liminf \operatorname{in}_{n \rightarrow \infty}\left\|\nabla u_{k}^{n}\right\|_{L^{p(x)}(\Omega)} \geq\left\|\nabla u_{k}\right\|_{L^{p(x)}(\Omega)}$. Therefore $\left\|\nabla u_{k}\right\|_{L^{p(x)}(\Omega)} \leq d_{k} / C_{1} C_{2}$. By the definition of the sequence $\left\{u_{k}^{n}\right\}_{n=1}^{\infty}$ we also get $\left|u_{k}^{n}(x)\right| \leq d_{k}$. Since $\left\{u_{k}^{n}\right\}_{n=1}^{\infty}$ is convergent almost everywhere, we get $\left|u_{k}(x)\right| \leq d_{k}$. So $u_{k} \in X_{k}$. Since

$$
W_{0}^{1, p(x)}(\Omega) \ni x \rightarrow \int_{\Omega} \frac{a_{k}(x)}{p(x)}|\nabla u(x)|^{p(x)} d x \in R
$$

is convex and lower semicontinuous, it is weakly lower semicontinuous [4] and since $\lim _{n \rightarrow \infty} \int_{\Omega} F_{u}^{k}\left(x, u_{k}^{n}(x)\right) d x=\int_{\Omega} F_{u}^{k}\left(x, u_{k}(x)\right) d x$ we get $\liminf _{n \rightarrow \infty} J_{k}\left(u_{k}^{n}\right) \geq J_{k}\left(u_{k}\right)$. Thus $J_{k}$ is weakly lower semicontinuous on $X_{k}$ and $J_{k}\left(u_{k}\right)=\inf _{u \in X_{k}} J_{k}(u)$.

Since $u_{k} \in X_{k}$ we may take $v_{k} \in X_{k}^{d}$ such that (3.1) holds. By the Fenchel-Young inequality

$$
\int_{\Omega} \frac{a_{k}(x)}{p(x)}\left|\nabla u_{k}(x)\right|^{p(x)} d x \geq \int_{\Omega}\left(v_{k}(x) \nabla u_{k}(x)-\frac{1}{\left(a_{k}(x)\right)^{q(x) / p(x)}} \frac{1}{q(x)}\left|v_{k}(x)\right|^{q(x)}\right) d x
$$

and by a direct calculation we obtain that $J_{k}\left(u_{k}\right) \geq J_{k}^{D}\left(v_{k}\right)$. By (3.4) it follows that $J_{k}\left(u_{k}\right) \leq \inf _{v \in X_{k}^{d}} J_{k}^{D}(v) \leq J_{k}^{D}\left(v_{k}\right)$. Hence $J_{k}\left(u_{k}\right)=J_{k}^{D}\left(v_{k}\right)$ and by a direct calculation we have

$$
\int_{\Omega} \frac{a_{k}(x)}{p(x)}\left|\nabla u_{k}(x)\right|^{p(x)} d x=\int_{\Omega}\left(v_{k}(x) \nabla u_{k}(x)-\frac{1}{\left(a_{k}(x)\right)^{q(x) / p(x)}} \frac{1}{q(x)}\left|v_{k}(x)\right|^{q(x)}\right) d x .
$$

Thus we actually have equalities in (3.6). Therefore (3.2) holds. Assertion (3.3) follows by (3.4) and since $J_{k}^{D}\left(v_{k}\right)=J_{k}\left(u_{k}\right)$. 
REMARK 1. From the proof of Theorem 3.1 it follows that the minimizing sequence $\left\{u_{k}^{n}\right\}_{n=1}^{\infty}$ has the following properties for $n \rightarrow \infty$ :

$u_{k}^{n} \rightarrow u_{k}$ weakly in $W_{0}^{1, p(x)}(\Omega)$,

$a_{k}(\cdot)\left|\nabla u_{k}^{n}(\cdot)\right|^{p(x)-2} \nabla u_{k}^{n}(\cdot) \rightarrow a_{k}(\cdot)\left|\nabla u_{k}(\cdot)\right|^{p(x)-2} \nabla u_{k}(\cdot)$ weakly in $L^{q(x)}(\Omega)$,

$\nabla u_{k}^{n} \rightarrow \nabla u_{k}$ weakly in $L^{p(x)}(\Omega)$ and

$u_{k}^{n} \rightarrow u_{k}$ a.e. in $\Omega$.

COROLlaRY 3.2. Assume Assumptions $1-3$ hold. For all $k=0,1,2, \ldots$ there exists $u_{k} \in X_{k}$ such that

$$
\begin{aligned}
& -\operatorname{div}\left(a_{k}(x)\left|\nabla u_{k}(x)\right|^{p(x)-2} \nabla u_{k}(x)\right)=F_{u}^{k}\left(x, u_{k}(x)\right),\left.\quad u_{k}\right|_{\partial \Omega}=0 \quad \text { and } \\
& J_{k}\left(u_{k}\right)=\inf _{u \in X_{k}} J_{k}(u) .
\end{aligned}
$$

Moreover $-\operatorname{div}\left(a_{k}(\cdot)\left|\nabla u_{k}(\cdot)\right|^{p(x)-2} \nabla u_{k}(\cdot)\right) \in L^{\infty}(\Omega)$ and $\left\|\nabla u_{k}\right\|_{L^{p(x)}(\Omega)} \leq d_{k} / C_{1} C_{2}$, $\left|u_{k}(x)\right| \leq d_{k}$.

Now we make an additional assumption on $p$.

Assumption 4. The region $\Omega$ has a Lipschitz-continuous boundary and for all $x, y \in \Omega$ such that $|x-y|<1$, we have

$$
|p(x)-p(y)|<\frac{M}{\ln (1 /|x-y|)}
$$

for a certain constant $M>0$.

This assumption guarantees that $C_{0}^{\infty}(\Omega)$ is dense in $W^{1, p(x)}(\Omega)$. Exactly as in [8] we prove the following result.

THEOREM 3.3. Assume Assumptions $1-4$ hold and that for all $u \in X_{0}$ there exists a subsequence $\left\{k_{i}\right\}_{i=1}^{\infty}$ such that $\lim _{i \rightarrow \infty} F_{u}^{k_{i}}(x, u(x))=F_{u}^{0}(x, u(x))$ weakly in $L^{q(x)}(\Omega)$. For each $k=0,1,2, \ldots$ there exists a solution $u_{k}$ to problem (1.1). There exists a subsequence $\left\{u_{k_{n}}\right\}_{n=1}^{\infty}$ of the sequence $\left\{u_{k}\right\}_{k=1}^{\infty}$ and $\bar{u} \in W_{0}^{1, p(x)}$ such that

$$
\begin{aligned}
& u_{k_{n}}-\bar{u} \in X, \quad \text { weakly in } W_{0}^{1, p(x)}(\Omega) \quad \text { and } \\
& -\operatorname{div}\left(a_{0}(x)|\nabla \bar{u}(x)|^{p(x)-2} \nabla \bar{u}(x)\right)=F_{u}^{0}(x, \bar{u}(x)),\left.\quad \bar{u}(x)\right|_{\partial \Omega}=0 .
\end{aligned}
$$

REMARK 2. From the proof contained in [8] it follows that the sequence $\left\{u_{k}\right\}_{k=1}^{\infty}$ that approximates $\bar{u}$ has the following properties for $k \rightarrow \infty$ :

$u_{k} \rightarrow \bar{u}$ weakly in $W_{0}^{1, p(x)}(\Omega)$,

$a_{k}(\cdot)\left|\nabla u_{k}(\cdot)\right|^{p(x)-2} \nabla u_{k}(\cdot) \rightarrow a_{0}(\cdot)|\nabla \bar{u}(\cdot)|^{p(x)-2} \nabla \bar{u}(\cdot)$ weakly in $L^{q(x)}(\Omega)$,

$\nabla u_{k} \rightarrow \nabla \bar{u}$ weakly in $L^{p(x)}(\Omega)$ and

$u_{k} \rightarrow \bar{u}$ a.e. in $\Omega$. 


\section{Approximation of solutions}

Following [9] the authors in [2] give finite-dimensional Galerkin-type approximation in the anisotropic case with suitable modification due to the properties of Orlicz-Sobolev spaces. In the case of Equation (1.4) we obtain, following reasoning from [2], that there exists a sequence $\left\{v_{k}^{n, j}\right\}_{j=1}^{\infty}$ of Galerkin approximations such that for a minimising sequence $\left\{u_{k}^{n}\right\}_{n=1}^{\infty}$ of $J_{k}$ over $X_{k}$ we have

$v_{k}^{n, j} \rightarrow u_{k}^{n}$ weakly in $W_{0}^{1, p(x)}(\Omega)$,

$a_{k}(\cdot)\left|\nabla v_{k}^{n, j}(\cdot)\right|^{p(x)-2} \nabla v_{k}^{n, j}(\cdot) \rightarrow a_{k}(\cdot)\left|\nabla u_{k}^{n}(\cdot)\right|^{p(x)-2} \nabla u_{k}^{n}(\cdot)$ weakly in $L^{q(x)}(\Omega)$,

$\nabla v_{k}^{n, j} \rightarrow \nabla u_{k}^{n}$ weakly in $L^{p(x)}(\Omega)$ and

$v_{k}^{n, j} \rightarrow u_{k}^{n}$ a.e. in $\Omega$.

THEOREM 4.1. Assume Assumptions 1-4 hold. For all $k=0,1,2 \ldots$ there exists $u_{k} \in X_{k}$ such that $J_{k}\left(u_{k}\right)=\inf _{u \in X_{k}} J_{k}(u)$ and

$$
-\operatorname{div}\left(a_{k}(x)\left|\nabla u_{k}(x)\right|^{p(x)-2} \nabla u_{k}(x)\right)=F_{u}^{k}\left(x, u_{k}(x)\right), \quad u_{k} \in W_{0}^{1, p(x)}(\Omega) .
$$

There exists a minimizing sequence $\left\{u_{k}^{n}\right\}_{n=1}^{\infty}$ for a restriction of a functional $J_{k}$ to the set $X_{k}$ such that $u_{k}^{n} \rightarrow u_{k}$ weakly in $W_{0}^{1, p(x)}(\Omega)$. Moreover there exists a sequence $\left\{v_{k}^{n, j}\right\}_{j=1}^{\infty}$ of finite-dimensional Galerkin approximations such that

$v_{k}^{n, j} \rightarrow u_{k}$ weakly in $W_{0}^{1, p(x)}(\Omega)$,

$a_{k}(\cdot)\left|\nabla v_{k}^{n, j}(\cdot)\right|^{p(x)-2} \nabla v_{k}^{n, j}(\cdot) \rightarrow a_{k}(\cdot)\left|\nabla u_{k}(\cdot)\right|^{p(x)-2} \nabla u_{k}(\cdot)$ weakly in $L^{q(x)}(\Omega)$,

$\nabla v_{k}^{n, j} \rightarrow \nabla u_{k}$ weakly in $L^{p(x)}(\Omega)$ and

$v_{k}^{n, j} \rightarrow u_{k}^{n}$ a.e. in $\Omega$.

PROOF. The existence of $u_{k} \in X_{k}$ and of a minimizing sequence $\left\{u_{k}^{n}\right\}_{n=1}^{\infty}$, having properties (3.5) and (1.4) follows by Theorem 3.1. By the remarks preceding the proof, for each $u_{k}^{n}$ for $n=1,2, \ldots$ there exists a sequence $\left\{v_{k}^{n, j}\right\}_{j=1}^{\infty}$ of Galerkin approximations such that

$v_{k}^{n, j} \rightarrow u_{k}^{n}$ weakly in $W_{0}^{1, p(x)}(\Omega)$,

$a_{k}(\cdot)\left|\nabla v_{k}^{n, j}(\cdot)\right|^{p(x)-2} \nabla v_{k}^{n, j}(\cdot) \rightarrow a_{k}(\cdot)\left|\nabla u_{k}^{n}(\cdot)\right|^{p(x)-2} \nabla u_{k}^{n}(\cdot)$ weakly in $L^{q(x)}(\Omega)$,

$\nabla v_{k}^{n, j} \rightarrow \nabla u_{k}^{n}$ weakly in $L^{p(x)}(\Omega)$ and

$v_{k}^{n, j} \rightarrow u_{k}^{n}$ a.e. in $\Omega$.

Now by Remark 1 we take a diagonal sequence with the property (4.1). 
Using Remark 2, by taking the diagonal sequence we obtain the following stability and approximation theorem.

THEOREM 4.2. Assume Assumptions $1-4$ hold and that for all $u \in X_{0}$ there exists a subsequence $\left\{k_{i}\right\}_{i=1}^{\infty}$ such that $\lim _{i \rightarrow \infty} F_{u}^{k_{i}}(x, u(x))=F_{u}^{0}(x, u(x))$ weakly in $L^{q(x)}(\Omega)$. For each $k=0,1,2, \ldots$ there exists a solution $u_{k}$ to Problem (1.1). There exists a subsequence $\left\{u_{k_{n}}\right\}_{n=1}^{\infty}$ of the sequence $\left\{u_{k}\right\}_{k=1}^{\infty}$ and $\bar{u} \in W_{0}^{1, p(x)}$ such that

$$
\begin{aligned}
& u_{k_{n}}-\bar{u} \in X, \text { weakly in } W_{0}^{1, p(x)}(\Omega) \quad \text { and } \\
& -\operatorname{div}\left(a_{0}(x)|\nabla \bar{u}(x)|^{p(x)-2} \nabla \bar{u}(x)\right)=F_{u}^{0}(x, \bar{u}(x)),\left.\quad \bar{u}(x)\right|_{\partial \Omega}=0 .
\end{aligned}
$$

Moreover there exists a sequence $\left\{v^{j}\right\}_{j=1}^{\infty}$ of finite-dimensional Galerkin approximations such that

$$
\begin{aligned}
& v^{j} \rightarrow \bar{u} \text { weakly in } W_{0}^{1, p(x)}(\Omega), \\
& a_{k}(\cdot)\left|\nabla v^{j}(\cdot)\right|^{p(x)-2} \nabla v^{j}(\cdot) \rightarrow a_{0}(\cdot)|\nabla \bar{u}(\cdot)|^{p(x)-2} \nabla \bar{u}(\cdot) \text { weakly in } L^{q(x)}(\Omega), \\
& \nabla v^{j} \rightarrow \nabla \bar{u} \text { weakly in } L^{p(x)}(\Omega) \quad \text { and } \\
& \dot{v}^{j} \rightarrow \bar{u} \text { a.e. in } \Omega \text {. }
\end{aligned}
$$

\section{References}

[1] R. A. Adams, Sobolev Spaces (Academic Press, New York, 1975).

[2] S. N. Antontsev and S. I. Shmarev, "Elliptic equations and systems with nonstandard growth conditions: existence, uniqueness and localization properties of the solutions", Nonlinear Anal. 65 (2006) 728-761.

[3] J. Chabrowski and Y. Fu, "Existence of solutions for $p(x)$-Laplacian problem on a bounded domain", J. Math. Anal. Appl. 306 (2005) 604-618.

[4] I. Ekeland and R. Temam, Convex Analysis and Variational Problems (North-Holland, Amsterdam, 1976).

[5] X. L. Fan and H. Zhang, "Existence of solutions for $p(x)$-Laplacian Dirichlet problem", Nonlinear Anal. 52 (2003) 1843-1852.

[6] X. L. Fan and D. Zhao, "On the spaces $L^{p(x)}(\Omega)$ and $W^{k, p(x)}(\Omega)$ ", J. Math. Anal. Appl. $263(2001)$ $424-446$.

[7] X. L. Fan and D. Zhao, "Sobolev embedding theorems for spaces $W^{k, p(x)}(\Omega)$ ", J. Math. Anal. Appl. 262 (2001) 749-760.

[8] M. Galewski, "On the existence and stability of solutions for Dirichlet problem with $p(x)$ Laplacian", J. Math. Anal. Appl. 326 (2007) 352-362.

[9] O. A. Ladyzhenskaya and N. N. Ural'tseva, Linear and quasi-linear elliptic equations (Academic Press, New York, 1968).

[10] M. Růžička, Electrorheological fluids: Modelling and Mathematical Theory, Volume 1748 of Lecture Notes in Mathematics (Springer-Verlag, Berlin, 2000).

[11] V. V. Zhikov, "Averaging of functionals of the calculus of variations and elasticity theory", Math. USSR Izv. 29 (1987) 33-66. 